\title{
Stratégies d'apprentissage des étudiants de premier cycle des études médicales, de graduat en biologie médicale et d'élèves infirmiers: une étude conduite au pôle universitaire européen Bruxelles-Wallonie.
}

\author{
Learming strategies of undergraduate students in medicine, medical biology and nusing school. \\ A study made at the "Pôle universitaire européen Bruxelles-Wallonie"
}

Nathalie VANMUYLDER ${ }^{1,2}$, Patrick SALVIA ${ }^{1}$, Françoise DE BROEU ${ }^{2}$, Marcel ROOZE ${ }^{1}$ et Stéphane LOURYAN ${ }^{1,3}$

Résumé But : Comparer les stratégies d'apprentissages mises en ceuvre par les étudiants en médecine de l'ULB au cours des 3 années du premier cycle des études médicales, les étudiants de première année de graduat en biologie médicale et les étudiants de l'école d'infirmier(e)s. Méthodes : A partir d'un référentiel connu, les stratégies d'apprentissages de 152 étudiants de premier cycle des études médicales, de 11 étudiants de $2^{2}$ candidature en kinésithérapie et éducation physique, de 44 étudiants de graduat et de 88 élèves-infirmières sont évaluées. Résultats : Une première approche a consisté à regrouper les cotations des outils de la " compréhension/appropriation " et de "l'analyselsynthèse " sous le label d'apprentissage en profondeur. Les scores moyens sont de 15,3 sur 20 en $1^{\text {re }}$ (CM1), 15,5 en $2^{\circ}$ et 14,8 en $3^{\circ}$ année de médecine, de 13,8 /20 en graduat (BM1) et de 14,1 chez les élèves-infirmières. Au cours des années successives de PCEM, la mémorisation diminue alors que la notion de relation augmente. Si les difficultés rencontrées diminuent en fonction de l'augmentation de confiance en soi, on observe des difficultés croissantes dans la gestion du temps, probablement en raison de la multiplication des cours. Pour l'apprentissage en profondeur, les outils qui semblent de moins en moins bien utilisés sont la reformulation et la réorganisation du travail, qui font appel à la synthèse sur laquelle l'enseignement universitaire de type transmissif ninsiste peut-être pas assez. Pour la comparaison BM1/CM1, à l'exception de la mémorisation, tous les outils évalués, quils se rapportent à des comportements cognitifs, métagognitifs ou affectifs, sont plus faiblement utilisés par les étudiants de BM1 que par ceux de CM1. Conclusions : Ces résultats encouragent les enseignants à recourir à des pédagogies différenciées, ce qui n'exclut pas des possibilités de recoupements.

Mots-clés Apprentissage ; stratégies ; stratégies d'apprentissage ; étudiants ; médecine ; biologie médicale ; soins infirmiers.

Summary Aim: To compare learning strategies of undergraduate medical students during their first 3 years of formation at the ULB with those of first year undergraduate students of medical biology and nursing school. Methods: With help of a referential, learning strategies were evaluated from 152 medical students (first three years), 11 second year students in physiotherapy, 44 first year students in medical biology and 88 first year students in nursing. Results: The comprehensionlappropriation and analysis/synthesis tools were merged in the category "deep learning". Mean scores for undergraduate medical students were 15.3/20 for the first year, 15.5/20 for the second year and 14.8/20 for the third year. Mean scores for first year undergraduate students in medical biology and nursing reached 13.8/20 and 14.1/20, respectively. During medicine formation, as years follow, memorization decreases, whereas associative knowledge increases. Difficulties diminished in correlation with a better self confidence but time management problems enhanced, perhaps because of a much more important amount of courses in the schedule. In connection with deep learning, the less used tools were reformulation and work reorganization, which both implicate global thinking ability, perhaps less solicited with teaching transmitting knowledge. With the exception of memorization, all cognitive, metacognitive and affective tools were less used from medical biology students as compare with medical students. Conclusions : These results support teachers to use different pedagogical approaches, but common strategies must not be excluded.

Key words Learning; strategies; learning strategies; students; medicine; medical biology; nursing. Pédagogie Médicale 2006;7:7-19 


\section{Recherche et Perspectives}

\section{Introduction}

\section{Problématique}

Depuis que le paradigme cognitiviste ${ }^{1}$ s'est imposé $^{\prime}$ comme une référence incontournable dans le champ de l'enseignement supérieur, la réflexion relative aux stratégies d'apprentissage domine le débat relatif à la réussite à l'université 2 .

Aux études supérieures, nombreux sont les étudiants qui éprouvent des difficultés à étudier efficacement ${ }^{3}$. Plusieurs résultats de recherches effectuées en Amérique du Nord confirment que les étudiants qui obtiennent des résultats académiques brillants ont un meilleur bagage stratégique que les étudiants faibles ${ }^{4,5}$. Le but d'améliorer la réussite scolaire des étudiants rend, dès lors, légitime que les enseignants s'intéressent à leurs stratégies d'apprentissage, sans omettre qu'une meilleure perception des outils de travail utilisés par les étudiants ne peut qu'aider l'enseignant dans sa tâche fondamentale, faire comprendre un savoir.

\section{Objectifs de l'étude}

L’objectif général de ce travail est donc de rapporter les résultats d'une enquête réalisée lors d'un programme d'évaluation-formation aux stratégies d'apprentissage, organisé à l'intention d'étudiants en sciences de la santé.

De façon spécifique, après avoir vérifié si les constatations relatives aux stratégies d'apprentissage des étudiants universitaires de psychologie restent valables en médecine et observé les variations possibles, l'objectif principal de cette étude a été d'établir les processus d'apprentissages des étudiants de graduats (enseignement supérieur de type court) en biologie médicale ou de l'école d'infirmier(ère)s et de comparer les résultats à ceux obtenus à l'université au cours de la première année du premier cycle des études médicales (PCEM). Un objectif complémentaire a été d'observer l'évolution des stratégies d'apprentissage mises en œuvre par les étudiants en médecine de l'Université Libre de Bruxelles (ULB) au cours des trois années du PCEM.

\section{Contexte local}

Le pôle universitaire européen Bruxelles-Wallonie réunit autour de l'Université Libre de Bruxelles (ULB) plusieurs " hautes écoles ", chargées de l'enseignement supérieur hors-universitét. Au sein de cet ensemble, le "pôle biomédical " associe la faculté de médecine (responsable du doctorat en médecine - 7 ans-, de la licence en science dentaire -5 ans-, de la licence en sciences biomédicales -4 ans-) et plusieurs établissements ressortissant aux " hautes écoles » et assurant notamment l'enseignement des soins infirmiers ( 3 ans et 4 ans pour les accoucheuses) et le graduat en biologie médicale ( 3 ans).

La situation décrite ici est celle qui prévalait avant la réforme dite " de Bologne ", qui institue un premier cycle de trois ans appelé "Bachelor ", suivi d'un deuxième cycle ou "Master", comportant 4 ans en médecine. Les trois premières années des études de médecine portaient au moment de l'étude le nom de candidatures. La première est consacrée aux sciences de base (chimie, physique, mathématique, biologie, introduction à l'anatomie, épistémologie). La deuxième est consacrée à la biologie de l'homme «normal » et à ses outils d'étude (anatomie, biochimie, histologie, embryologie, physiologie, statistique, etc.). La troisième est consacrée à la suite de l'" homme normal » (anatomie, pharmacologie, physiologie, etc.) et à une introduction à la pathologie (sémiologie, anatomie pathologique, biochimie pathologique, etc.). Le paradigme pédagogique demeure celui du cours magistral mais le nombre d'heures de travaux pratiques est proportionnellement très élevé en première et deuxième candidatures, tandis qu'il se réduit en troisième année, alors même que le nombre de cours différents et d'examens y atteint son maximum (environ 20 épreuves).

\section{Matériel et méthodes}

\section{Evaluation des stratégies d'apprentissage déclarées des étudiants}

A partir d'un référentiel connu et validé 7 , les stratégies d'apprentissages déclarées des étudiants en médecine de l'ULB, de graduat en biologie médicale de la Haute Ecole Fransisco Ferrer (HEFF) et de graduat de l'Ecole d'infirmier(ère)s annexée à l'ULB (Haute Ecole Ilya Prigogine HEIP-) sont évaluées. Dans un premier temps, le choix d'une deuxième candidature a été fait pour permettre une comparaison entre notre échantillon et l'échantillon connu de deuxième candidature en science psychologique et de l'éducation (PSYCHO2) à l'ULB, qui constituait le groupe témoin de l'étude princeps, le questionnaire de départ ayant été réalisé pour une étude concernant l'enseignement secondaire. Par la suite, les premières et troisiè-

\footnotetext{
1- Laboratoire d'anatomie et d'embryologie - Faculté de médecine - Université Libre de Bruxelles (ULB) - 808 route de Lennik CP 619 - 1070 Bruxelles - Belgique

2- Unité d'enseignement et de recherche de biologie médicale - Haute école Francisco Ferrer - rue Terre-Neuve 116 -1000 Bruxelles 3- Cellule pédagogique de la faculté de médecine de l'ULB Correspondance : Stéphane Louryan - Laboratoire d'Anatomie et d'Embryologie - Faculté de Médecine - Université Libre de Bruxelles 808 route de Lennik - CP 619 -1070 Bruxelles - Belgique - mailto:Slouryan@ulb.ac.be
} 
mes candidatures en sciences médicales, puis les premières années de graduat en biologie médicale ont également été évaluées.

Brièvement, nous rappelons les compétences qui ont été évaluées, à savoir :

- les comportements d'ordre cognitif que les étudiants déclarent mettre en œuvre lors de leurs apprentissages (compréhension, analyse, synthèse, etc.) ;

- les activités métacognitives utilisées (planification du travail, auto-évaluation) ;

- les aspects affectifs et la motivation (projet d'avenir, confiance en soi).

Le questionnaire validé "Mes outils de travail intellectuels» ${ }^{7}$ a été repris quasi intégralement hormis la question 30 concernant «mon avenir ». Lensemble du questionnaire utilise des questions à réponses fermées (de type : jamais, rarement, souvent, toujours). La consistance interne du questionnaire avait été déterminée lors de l'étude d'origine (coefficient alpha de Cronbach $=0,82)^{7}$. La rubrique " apprentissage en profondeur " est constituée de la partie " compréhension-appropriation" (3 outils) et de la partie " analyse-synthèse » (3 outils). La rubrique " mémorisation » inclut la " mémorisation par cœur » et la " mémorisation appropriative ». Certains outils explorent la gestion du temps, les difficultés et des items liés à la réussite.

\section{Collectif}

Léchantillon étudié comprend : 56 étudiants de $2^{\mathrm{e}}$ candidature en médecine (CM2), 36 pour l'année académique 1998-1999 et 20 pour l'année 2000-2001; 77 étudiants de première candidature en médecine (CM1), soit 17 pour l'année académique 2001-2002 et 60 pour l'année 2003-2004; 19 étudiants de troisième candidature en médecine (CM3), 88 étudiants de première année d'études d'infirmier(ères)/accoucheur(euse)s (I/A1) et 44 étudiants de première année de graduat en biologie médicale (BM1), 24 pour l'année académique 2001-2002 et 20 pour 2003-2004. Nous avons demandé aux étudiants du PCEM et de l'école d'infirmier(ère)s de s'identifier; si un étudiant marquait une réticence, nous lui laissions le choix de la confidentialité. Pour des raisons purement administratives (à la demande de l'établissement d'enseignement), l'enquête concernant les étudiants de graduat en biologie médicale était complètement anonyme.

La répartition de l'échantillon étudié selon les genres s'établit comme suit : CM1: 22 filles, 10 garçons et 45 non identifiés ; CM2 : 18+11 (29) filles et 19+8 (27) garçons ; CM3 : 7 filles, 4 garçons et 8 non identifiés ; BM1: 19 filles, 10 garçons et 15 non identifiés ; I/A1 : 62 filles, 14 garçons et 12 non identifiés.

Pour les candidatures en médecine, les étudiants convo- qués au test appartenaient à un groupe de travaux pratiques (TP) pris au hasard ; par contre, tous les étudiants de graduats en biologie médicale et tous les étudiant(e)s infirmier(ère)s ont été invités à remplir le questionnaire.

\section{Administration du questionnaire et traitement des réponses}

Le questionnaire a été remis aux étudiants volontaires (50\% en moyenne de taux de participation) au début du second semestre, après la session d'examens de janvier, dans toutes les années et toutes les sections. Une explication sur le sens du questionnaire a été donnée, commentant son intérêt et la sincérité requise pour le remplir. Ce questionnaire a été rempli en auditoire, en environ 50 minutes.

Le dépouillement a consisté à retranscrire les différents items (outils) dans un tableur. Les statistiques descriptives (moyenne, déviation standard, maximum, minimum) ont été calculées. Les qualificatifs « jamais » et « rarement » ont été associés de même que les qualificatifs "souvent " et " toujours ». Une valeur de 0 a été attribuée pour le groupement « jamais-rarement » et de 1 pour celui « souventtoujours ". Le questionnaire ${ }^{7}$ est construit de telle manière que la fréquence d'utilisation des différents outils est calculée sous forme d'un score à partir du nombre de fois où la réponse "souvent-toujours " a été donnée aux questions répertoriées comme appartenant à l'outil en question. Le score repris dans les tableaux correspond à la moyenne des valeurs collectées dans la population concernée. L'analyse des réponses fournies au questionnaire est réalisée par les étudiants eux-mêmes, puis vérifiée lors de l'encodage des résultats.

Des comparaisons non statistiques ont été faites, d'une part, entre les résultats obtenus chez les étudiants de nos différents échantillons, d'autre part, entre les résultats obtenus respectivement chez nos étudiants de CM2 et chez ceux de PSYCHO2. Les données concernant le groupe $\mathrm{PSYCHO} 2$ sont celles récoltées auprès d'une centaine d'étudiants universitaires de $2^{\mathrm{e}}$ candidature en science psychologique et de l'éducation afin de servir de groupe témoin lors de l'étude princeps ${ }^{7}$. Dans ce précédent travail, le programme de cours de candidature en sciences psychologiques avait été considéré comme un témoin représentatif des exigences propres aux études universitaires? ${ }^{7}$. Le programme d'étude comprenant à la fois des cours de sciences bio-médicales (statistique, biologie, génétique, cytologie, histologie) et de sciences humaines proches de ceux rencontrés en candidature en médecine, il nous est apparu que ce groupe pouvait également servir de témoin pour notre étude.

Pour les étudiants de CM2 de 1998-1999, un coefficient 


\section{Recherche et Perspectives}

de corrélation a été calculé pour chacun des outils de travail relativement aux résultats obtenus par ces étudiants en première candidature en terme de grades. L'examen d'anatomie en CM1 est un examen de type "questionnaire à réponse ouverte courte " $(\mathrm{QROC})$, comptant huit questions, chacune cotée sur 10 par rapport à une grille de correction. Le résultat final est exprimé par un score sur 20. Un test du Khi-deux a été calculé entre les types de réponse au questionnaire (jamais-rarement et souventtoujours) et les résultats à l'examen d'anatomie de première candidature exprimés en deux groupes (score $>14$ sur 20 et score d'anatomie $\leq 14$ sur 20 ). Ce test vise à montrer si une relation existe entre les proportions d'étudiants répondant jamais ou rarement à un item du questionnaire et les proportions de bonnes réussites à l'examen d'anatomie.

Le nombre de réponses pour chacune des deux catégories a été exprimé en pourcentage.

La comparaison globale entre tous les groupes a fait l'objet d'une analyse statistique à l'aide du test de KruskalWallis, tandis que les comparaisons entre populations par groupe de deux ont fait l'objet d'un test de MannWhitney outil par outil. La limite de signification a été définie à $\mathrm{p} \leq 0,05$.

\section{Résultats}

Nous étant intéressés au questionnaire relatif aux stratégies d'apprentissage, une analyse transversale des résultats a été initialement établie. Les résultats de nos échantillons ont été comparés ensuite à ceux des étudiants en psychologie et science de l'éducation.

Afin de permettre la comparaison, les aspects cognitifs et métacognitifs sont présentés séparément des aspects plus affectifs.

\section{Etude comparative entre la CM2 (cohorte 1998-1999) et la PSYCHO2}

Les résultats bruts (collation des réponses au questionnaire "Mes outils de travail intellectuels") sont présentés dans le tablean 1.

\section{Apprentissage en profondeur}

Dans la typologie à laquelle nous nous référons, le concept d' " apprentissage en profondeur " fait référence à deux stratégies de traitement de l'information: la " compréhension-appropriation » et l'" analyse-synthèse ". - La " compréhension-appropriation » fait référence aux outils de travail intellectuels 1, 2 et 3 .
La moyenne des valeurs de notre échantillon, quant à la recherche de sens et d'informations, est 4,4 $(\mathrm{SD}=0,7)$ en CM2 vs 4,2 dans l'échantillon PSYCHO2 (Outils 1). La moyenne pour la reformulation est de 4,5 $(\mathrm{SD}=0,7)$ en $\mathrm{CM} 2$ vs 2 en PSYCHO2 (Outils 2). La moyenne pour l'exemplification est de 4,5 $(\mathrm{SD}=0,7)$ en $\mathrm{CM} 2$ vs 1,6 en PSYCHO2 (Outils 3).

Le total pour la " compréhension-appropriation " est donc de 8,3 en CM2E vs 7,8 en PSYCHO2.

- L' « analyse-synthèse " fait référence à l'outil 4 et ses deux items, l'un d'analyse (" analyser les relations") et l'autre de structuration (" faire un plan »). L'outil 5 fait appel à la réorganisation de l'information.

La moyenne pour la partie 1 est de 3,7 $(\mathrm{SD}=1,1)$ vs 3,7 en PSYCHO2. La moyenne pour la partie 2 est de 1,1 $(\mathrm{SD}=0,8)$ vs 1,2 en PSYCHO2. La moyenne pour l'outil 5 est 2,2 (SD $=0,9)$ vs 2,3 en PSYCHO2.

Le total pour l' " analyse-synthèse " est donc de 7,0 $(\mathrm{SD}=1,7)$ en $\mathrm{CM}$ vs 7,2 en PSYCHO2.

Un score de 15,3 sur 20 est obtenu dans notre échantillon vs 15 en PSYCHO2

Une valeur du coefficient de corrélation de 0,15 (p $=0,05)$ a été calculée entre les résultats de l'apprentissage en profondeur et les résultats scolaires obtenus en première candidature.

\section{Mémorisation}

Dans la typologie à laquelle nous nous référons, le concept de "mémorisation" fait référence aux outils " mémorisation par cour » et " mémorisation appropriative ", dans le cadre de l'application stricte du questionnaire-type $\mathrm{e}^{7}$.

- La " mémorisation par cœur " recueille une valeur moyenne de $0,6(\mathrm{SD}=0,7)$ en CM2 vs 1,1 en PSYCHO2. - Les " formes appropriatives de mémorisation " recueillent une valeur moyenne de $2,3(\mathrm{SD}=0,8)$, identique à la valeur retrouvée en $\mathrm{PSYCHO} 2$.

\section{Aspects affectifs}

- Nombre de difficultés rencontrées:

la valeur moyenne de 2,5 (SD = 2,3) en CM2 est inférieure à celle de 3,8 en PSYCHO2 Les sous-groupes " filles " et " garçons " montrent des valeurs de 2,8 et 2,4 respectivement.

- La confiance en ses capacités :

une valeur moyenne de 2,3 (SD 0,9) est observée dans le groupe CM1. Les sous-groupes filles " et "garçons " montrent des valeurs identiques $(2,3)$.

- Implication-engagement :

une valeur de 4,5 (SD 1,6) est observée dans le groupe $\mathrm{CM} 1$ vs 3,8 en PSYCHO2. 


\section{Tableau 1 :}

Valeurs moyennes $( \pm 1$ DS), maximales et minimales des scores d'utilisation des outils de travail intellectuels utilisés par les étudiants de $2^{\mathrm{e}}$ candidature en médecine (CM2) - cohorte 1998-1999 et en science psychologique et de l'éducation (PSYCHO2)

\begin{tabular}{|c|c|c|c|c|c|c|c|}
\hline & \multicolumn{6}{|c|}{$\mathrm{CM} 2$} & \multirow[t]{2}{*}{ PSYCHO 2} \\
\hline & Score & Moy. & DS & Max. & Min. & Corrélation & \\
\hline $\begin{array}{r}\text { Outil } 1 \text { : recherche de sens } \\
\text { et d'informations }\end{array}$ & 5 & 4,4 & 0,7 & 5,0 & 3,0 & $-0,19$ & 4,2 \\
\hline Outil 2 : reformulation & 3 & 2,2 & 0,7 & 3,0 & 0,0 & $-0,06$ & 2,0 \\
\hline Outil 3 : exemplification & 2 & 1,6 & 0,5 & 2,0 & 1,0 & 0,16 & 1,6 \\
\hline $\begin{array}{l}\text { Compréhension } \\
\text { Appropriation (outils } 1 \text { à 3) }\end{array}$ & 10 & 8,3 & 1,9 & 11,0 & 4,0 & $-0,09$ & 7,8 \\
\hline \multirow{3}{*}{$\begin{aligned} & \text { Outil } 4 \mathrm{~A} \text { : analyse des relations } \\
& \text { Outil } 4 \mathrm{~B} \text { : faire un plan } \\
& \text { Outil } 5 \text { : réorganisation } \\
& \text { de l'information }\end{aligned}$} & 5 & 3,7 & 1,1 & 5,0 & 1,5 & 0,31 & 3,7 \\
\hline & 2 & 1,1 & 0,8 & 2,0 & 0,0 & 0,15 & 1,2 \\
\hline & 3 & 2,2 & 0,9 & 3,0 & 0,0 & 0,14 & 2,3 \\
\hline Analyse-synthèse (outils 4 et 5) & 10 & 7,0 & 1,7 & 8,0 & 2,5 & 0,28 & 7,2 \\
\hline $\begin{array}{l}\text { Apprentissage en profondeur } \\
\text { (outils } 1 \text { et } 5 \text { ) }\end{array}$ & 20 & 15,3 & 2,4 & 18,0 & 10,0 & 0,15 & 15,0 \\
\hline Outil 6A : mémorisation par cœur & 3 & 0,6 & 0,7 & 3,0 & 0,0 & 0,14 & 1,1 \\
\hline $\begin{array}{c}\text { Outil 6B : mémorisation sous forme } \\
\text { appropriative }\end{array}$ & 3 & 2,3 & 0,8 & 3,0 & 1,0 & $-0,08$ & 2,3 \\
\hline Mémorisation (outil 6) & 6 & 2,9 & 0,9 & 4,0 & 1,0 & 0,04 & 3,4 \\
\hline $\begin{array}{c}\text { Outil } 7 \text { : anticipation des situations } \\
\text { d'évaluation }\end{array}$ & 4 & 2,5 & 1,1 & 4,0 & 0,0 & $-0,07$ & \\
\hline Outil 8 : gestion du temps & 4 & 2,6 & 1,3 & 4,0 & 0,0 & 0,35 & \\
\hline $\begin{array}{c}\text { Outil 9A : nombre de difficultés } \\
\text { rencontrées }\end{array}$ & 15 & 2,5 & 2,3 & 9,0 & 0,0 & $-0,16$ & 3,9 \\
\hline Outil 9B : confiance en soi & 3 & 2,3 & 0,9 & 3,0 & 0,0 & 0,13 & 2,1 \\
\hline Outil 10 : implication-engagement & 7 & 4,5 & 1,6 & 7,0 & 1,0 & 0,27 & 3,8 \\
\hline $\begin{array}{l}\text { Items liés à la réussite } \\
\text { (outils } 1 \text { à } 5,7,8,9 \mathrm{~B} \text { et } 10 \text { ) }\end{array}$ & 38 & 26,1 & 4,7 & 34,5 & 17,0 & 0,28 & \\
\hline
\end{tabular}

Les corrélations sont calculées entre ces valeurs et les résultats obtenus par ces mêmes étudiants en $1^{r e}$ candidature 


\section{Recherche et Perspectives}

\section{Analyse par dimension}

- Le score total pour la "compréhension-appropriation" est de 8,3 sur 10 et celui pour l'" analyse-synthèse " de 7,0 sur 10 .

- Le "sentiment de faible compétence " recueille une valeur moyenne de 2,5 pour l'outil 9A. Remarquons que les pourcentages de réponse "souvent-toujours " aux items 14.1 (anciennement 36.1) et 34.5 sont de $76 \%$ pour ces deux questions. L'item 4.5 (quand je rate un examen, c'est souvent dû à une mauvaise manière d'étudier) recueille une réponse affirmative dans $51 \%$ des cas.

- "L'implication ou engagement " recueille une valeur de 4,5 sur 10 en moyenne avec des maxima à 7 pour l'outil 10.

Sur 5 étudiants ayant obtenu une "grande distinction" en CM1, 4 ont des valeurs entre 6 et 7 sur 10 . Une des étudiantes ayant une grande distinction obtient une valeur de 4 et l'on remarque que, pour cette étudiante, l'ensemble des résultats paraît paradoxal.

Nous avons observé les pourcentages obtenus pour chacun des items retenus. Pour l'item 4.2 (lorsque je rate un examen, c'est souvent dû à un manque d'intérêt pour le sujet), le pourcentage de «jamais-rarement » est $76 \%$. Pour l'item 4.1 (lorsque je rate un examen, c'est souvent dû à un manque d'étude), ce pourcentage est de $54 \%$. Pour l'item 14.2 (je me laisse facilement décourager), le pourcentage de « jamais-rarement » est de $78 \%$. Pour l'item 16 (j'ai tendance à facilement me déconcentrer lors de mon étude), le pourcentage de « jamais-rarement » est de $41 \%$. Pour l'item 18.2 (face à un exercice difficile, j'essaie de le faire à tout prix), le pourcentage de " souvent- toujours » est de $76 \%$. Pour l'item 38.2 (lorsque je présente un examen, je vais essayer d'avoir les meilleurs résultats possibles), le pourcentage de réponses affirmatives est de $46 \%$. Pour l'item 41.3 ou 4 (concentration sans interruption de 60 minutes et plus), le pourcentage de réponses affirmatives est de $68 \%$. Les réponses concernant l'item 16 et l'item 4.1 semblent contradictoires. L'item 38.2 a montré 17 réponses affirmatives pour 16 réponses affirmatives à l'item 38.3 (je fais de mon mieux, peu importe les résultats).

- L'" anticipation des situations d'évaluation et la gestion du temps", estimées avec les nouveaux outils 7 et 8 recueillent des valeurs moyennes de 2,5 et 2,6 sur 4 (Tableau 1). Une corrélation a été calculée entre les résultats de ces outils et l'outil 10 ainsi que les notes de CM1. Ces coefficients de corrélation sont donnés dans le tableau 2. Une corrélation semble s'établir entre l'outil de gestion du temps et l'outil d'implication/engagement.

\section{Tableau 2 :}

Coefficients de corrélation entre les scores d'utilisation des outils 7 et 8 et le score d'utilisation de l'outil 10 ou le score aux examens de première année (CM1), établis chez les étudiants de $2^{\mathrm{e}}$ candidature en médecine.

\begin{tabular}{|c|c|c|}
\hline & Outil 7 & Outil 8 \\
\hline Outil 10 & 0,06 & 0,39 \\
\hline Note CM1 & $-0,06$ & 0,35 \\
\hline
\end{tabular}

\section{Comparaison des stratégies d'apprentissage mises en cuvre par les étudiants en médecine de l'ULB au cours des 3 années du PCEM}

Les résultats bruts sont présentés dans le tableau 3.

Sur la figure 1, nous avons rapporté toutes les notations de chacun des outils à un score sur 20. Afin de tenter de quantifier les résultats, une première approche a consisté à regrouper les scores des outils de la " compréhension-appropriation » et de "l'analyse/synthèse » sous le label proposé de l'apprentissage en profondeur. Ensuite, nous leur avons ajouté celles qui sont liées à la gestion du temps et aussi celles faisant intervenir un engagement, une implication. Lors de la transition entre la première et la deuxième année, nous remarquons une augmentation statistiquement significative de l'utilisation des outils relations, analyse-synthèse, et gestion du temps. En revanche, lors du passage en troisième année, on déplore une chute de l'analyse-synthèse et de la mémorisation par appropriation. L'apprentissage en profondeur ne se modifie pas, tandis que l'outil mémorisation décroît également.

\section{Comparaison des stratégies d'apprentissage mises en cuvre par les étudiants de CM1 de l'ULB, de BM1 de l'HEFF et de I/A1 de l'ULB/HEIP}

Les données brutes sont présentées sur le tableau 4.

Sur la figure 2, nous avons rapporté toutes les notations de 


\section{Tableau 3 :}

Valeurs moyennes des scores d'utilisation des outils de travail intellectuels utilisés par les étudiants de $1^{\text {re }}(\mathrm{CM} 1), 2^{\mathrm{e}}(\mathrm{CM} 2)$ et $3^{\mathrm{e}}(\mathrm{CM} 3)$ années de candidature en médecine et chez les étudiants de $2^{\mathrm{e}}$ année de science psychologique et de l'éducation (PSYCHO2)

\begin{tabular}{|c|c|c|c|c|c|}
\hline & Score & CM1 & CM2 & CM3 & PSYCHO2 \\
\hline Outil 1 : recherche de sens et d'informations & 5 & 4,5 & 4,5 & 4,5 & 4,2 \\
\hline Outil 2 : reformulation & 3 & 2,1 & 2,2 & 1,8 & 2,0 \\
\hline Outil 3 : exemplification & 2 & 1,7 & 1,7 & 1,6 & 1,6 \\
\hline $\begin{array}{l}\text { Compréhension } \\
\text { Appropriation (outils } 1 \text { à 3) }\end{array}$ & 10 & 8,3 & 8,4 & 7,9 & 7,8 \\
\hline Outil 4A : analyse des relations & 5 & 3,2 & 3,8 & 4,0 & 3,7 \\
\hline Outil $4 \mathrm{~B}$ : faire un plan & 2 & 1,3 & 1,4 & 1,7 & 1,2 \\
\hline $\begin{aligned} \text { Outil 5: } & \text { réorganisation } \\
& \text { de l'information }\end{aligned}$ & 3 & 2,4 & 1,9 & 1,2 & 2,3 \\
\hline Analyse-synthèse (outils 4 et 5) & 10 & 7,0 & 7,1 & 6,9 & 7,2 \\
\hline $\begin{array}{l}\text { Apprentissage en profondeur } \\
\text { (outils } 1 \text { et } 5)\end{array}$ & 20 & 15,3 & 15,5 & 14,8 & 15,0 \\
\hline Outil 6A : mémorisation par cœur & 3 & 0,7 & 0,5 & 0,2 & 1,1 \\
\hline $\begin{array}{l}\text { Outil 6B : mémorisation sous forme } \\
\text { appropriative }\end{array}$ & 3 & 2,5 & 2,5 & 2,2 & 2,3 \\
\hline Mémorisation (outil 6) & 6 & 3,1 & 3,0 & 2,4 & 3,4 \\
\hline $\begin{array}{c}\text { Outil } 7 \text { : anticipation des situations } \\
\text { d'évaluation }\end{array}$ & 4 & 2,4 & 2,4 & 2,3 & \\
\hline Outil 8 : gestion du temps & 4 & 2,3 & 2,3 & 2,3 & \\
\hline Outil 9A : nombre de difficultés rencontrées & 15 & 4,5 & 3,0 & 2,2 & 3,9 \\
\hline Outil 9B : confiance en soi & 3 & 1,9 & 2,2 & 2,3 & 2,1 \\
\hline Outil 10 : implication-engagement & 7 & 4 & 4,2 & 4,1 & 3,8 \\
\hline $\begin{array}{l}\text { Items liés à la réussite } \\
\text { (outils } 1 \text { à } 5,7,8,9 B \text { et } 10)\end{array}$ & 38 & 25,1 & 26,2 & 25,6 & \\
\hline
\end{tabular}




\section{Recherche et Perspectives}

\section{Tableau 4 :}

Valeurs moyennes des scores d'utilisation des outils de travail intellectuels utilisés par les étudiants de $1^{\text {re }}$ candidature en médecine (CM1), de $1^{\text {re }}$ année de graduat en études d'infirmiers(ères)/accouchers(euses) (I/A1) et en biologie médicale (BM1)

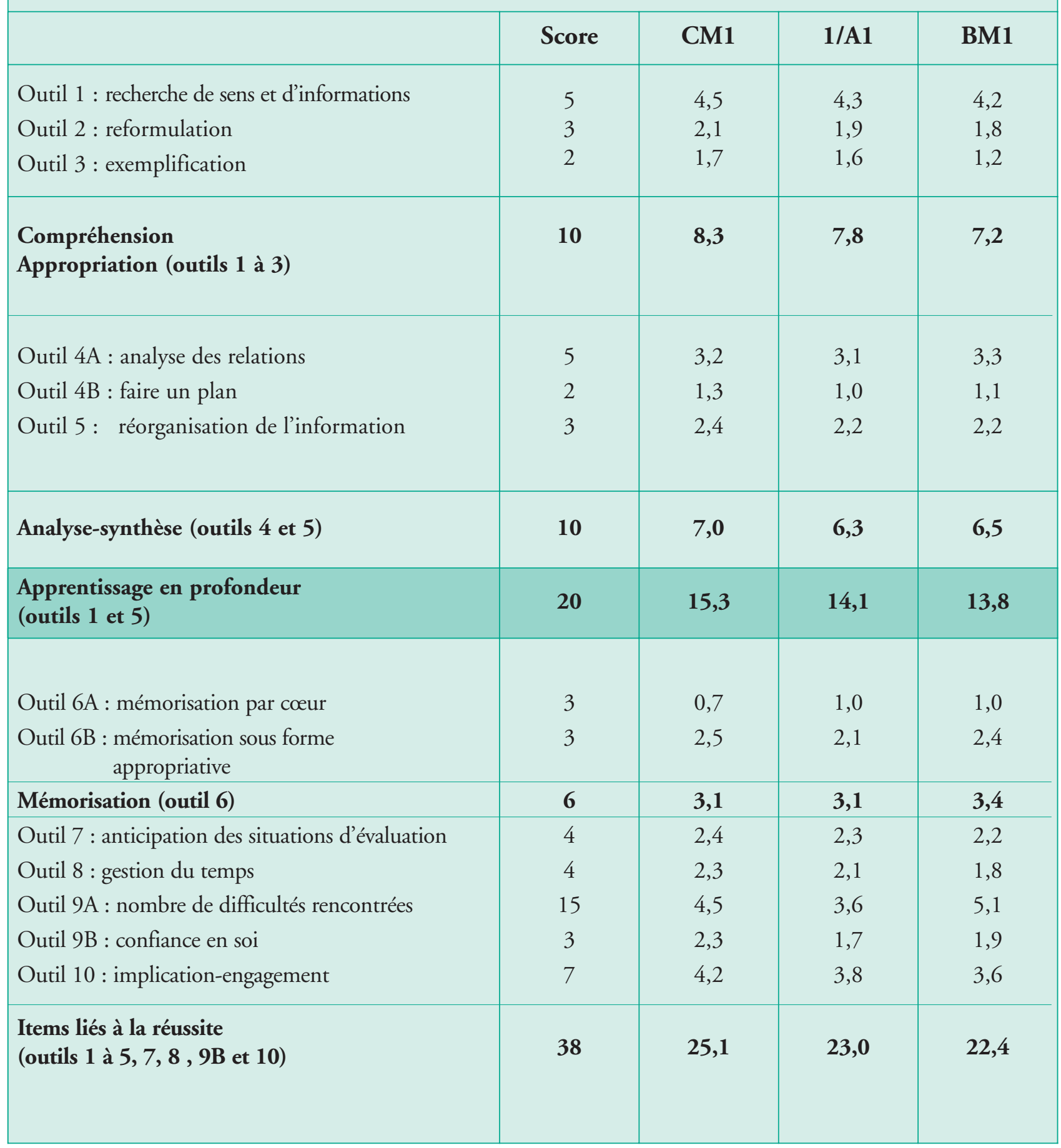




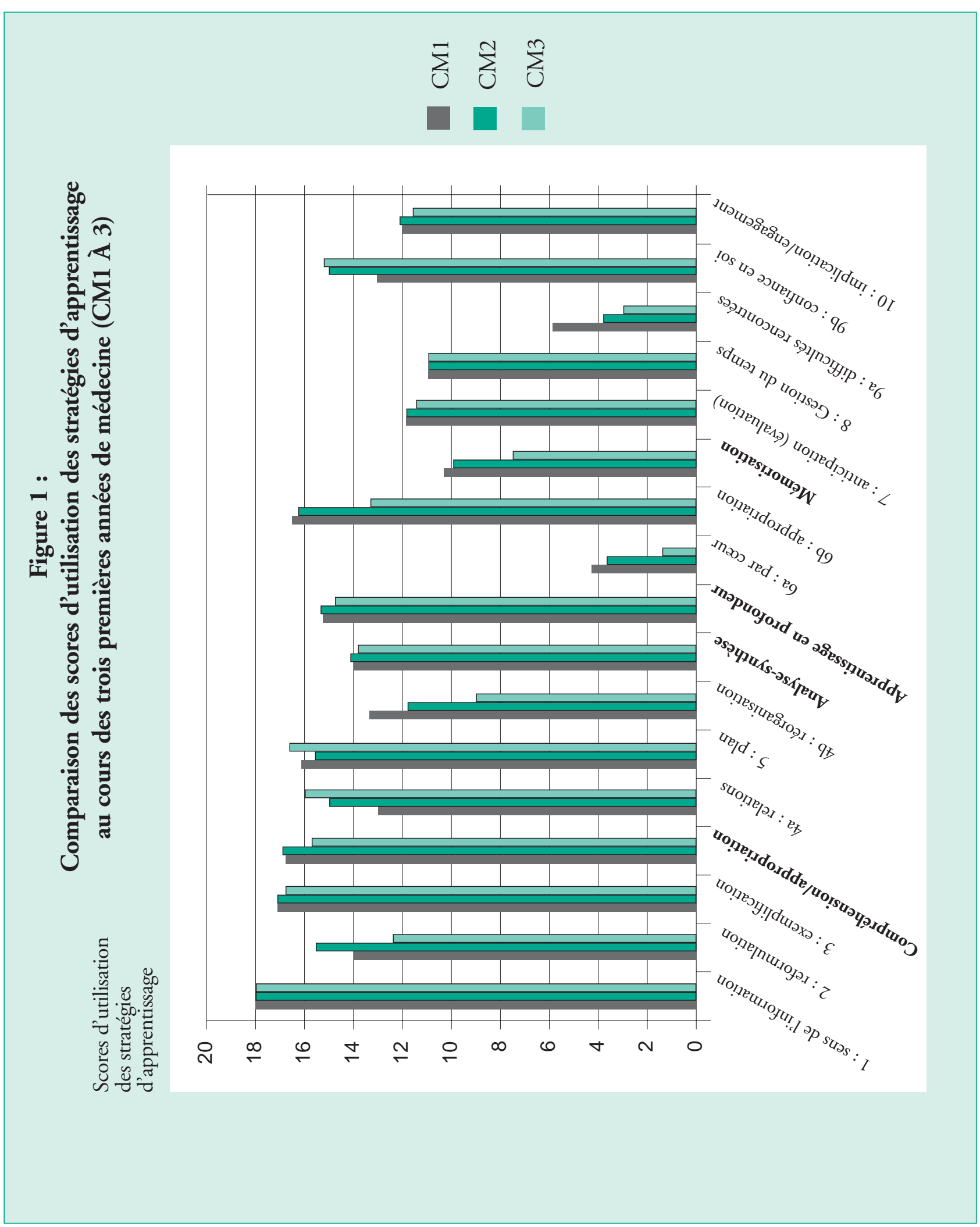




\section{Recherche et Perspectives}

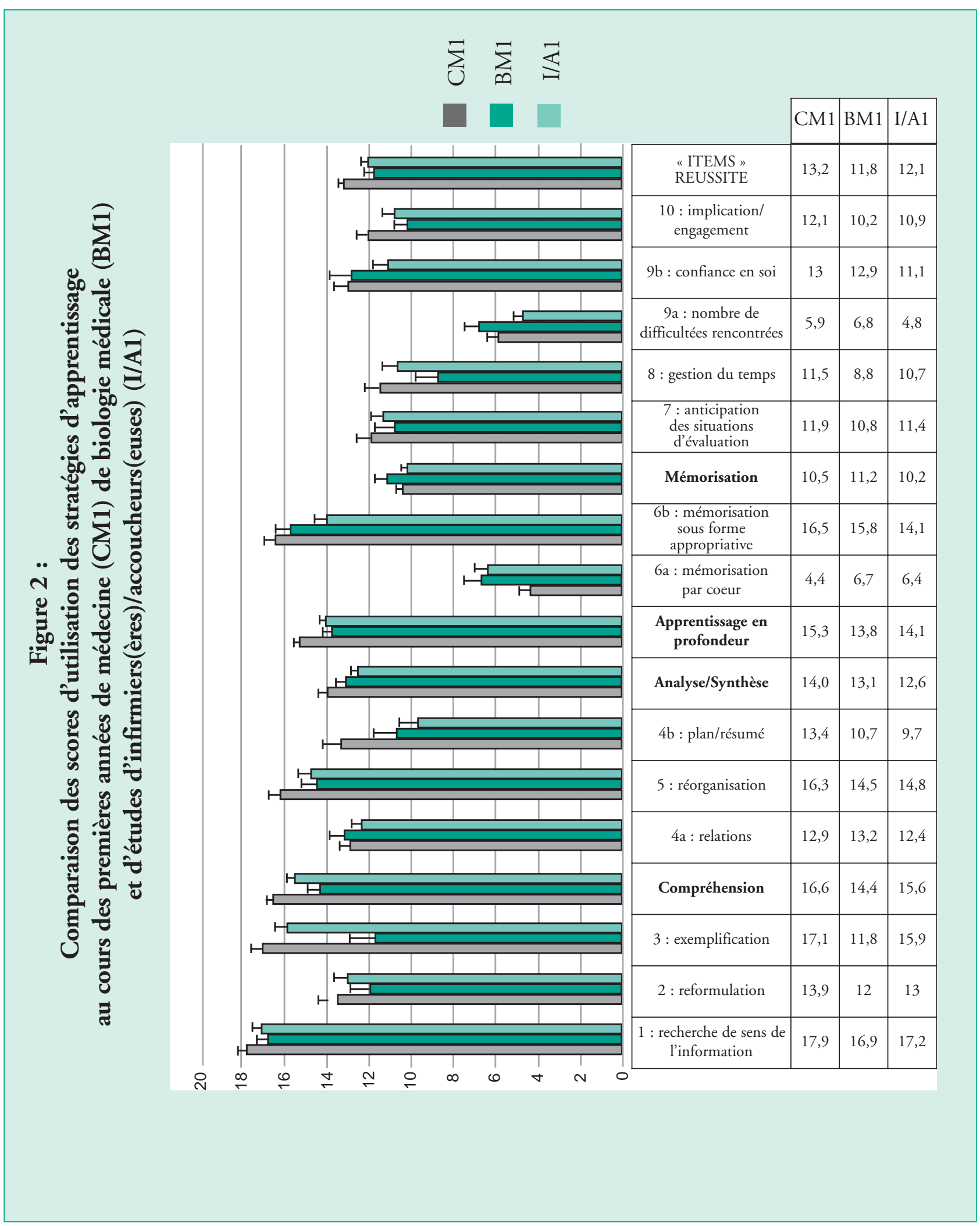


chacun des outils à un score sur 20. Comme précédemment, les scores des outils de la « compréhension/appropriation » et de "l'analyse/synthèse » ont été regroupées sous le label proposé de l'" apprentissage en profondeur ». Ensuite, nous leur avons ajouté celles qui sont liées à la " mémorisation ", à la " gestion du temps » ainsi que celles faisant intervenir un engagement, une implication. Enfin, nous avons regroupé sous le vocable « items réussite » l'ensemble des outils déterminés par l'étude de Wolfs comme intervenant sur la réussite scolaire ${ }^{7}$. On retiendra que la confrontation des étudiants de CM1 avec ceux de BM1 fait apparaître des résultats statistiquement significatifs en faveur des étudiants en médecine pour les outils « exemplification ", " compréhension-appropriation ", "planification ", " réorganisation ", " gestion du temps » et " implication-engagement». En revanche, l'outil « mémorisation par cœur » est significativement plus utilisé en BM1. La comparaison des étudiants en CM1 avec ceux de I/A1 montre des résultats similaires, à l'exception de l'outil « exemplification ", non significativement différent. La confrontation des étudiants en I/A1 avec ceux de BM1 montre assez peu de résultats significatifs : recours accru à l'" exemplification » et la " compréhension-appropriation » en faveur des étudiants de I/A1 et davantage de difficultés rencontrées par les étudiants en BM1.

\section{Discussion}

Les résultats de notre étude indiquent une similitude dans les approches d'apprentissage chez les étudiants universitaires de CM2 (cohorte 1998-1999) et de PSYCHO2. Le choix d'une stratégie d'apprentissage en profondeur semble établi. Un coefficient de corrélation significatif a été établi entre les recours aux stratégies d'" apprentissage en profondeur " et les résultats scolaires obtenus en CM1 : les étudiants qui utilisent le plus les outils de l'apprentissage en profondeur réussissent le mieux l'examen d'anatomie de première année. Il faut, cependant, noter le biais dû à l'échantillon d'étudiants qui de fait ont réussi leur première candidature.

Les résultats concernant les stratégies d'apprentissage utilisées par les étudiants de PCEM sont contrastés. Concernant l'" apprentissage en profondeur ", les outils qui semblent les moins bien et de moins en moins utilisés sont la reformulation et la réorganisation, qui font appel à la synthèse sur laquelle l'enseignement universitaire de type transmissif n'insiste peut-être pas assez. Nos questions d'évaluation font encore appel à un savoir magistral retransmis parfois de façon décousue par l'étudiant.

La " mémorisation par cœur" ne semble pas être une stratégie essentielle de l'étudiant en médecine mais il faut sans doute faire la part de la connotation négative de ce terme dans notre enseignement magistral. En effet, lors des examens la plupart des questionnaires favorisent la «mémorisation-appropriative " au détriment de la mémorisation «par cour » qui est généralement sanctionnée. De manière générale, l'usage de la " mémorisation " diminue alors que le recours à l' " analyse des relations " augmente. L'étudiant en médecine semble éprouver peu de difficultés (outil 9A d'auto-évaluation de ces capacités). De plus, si les difficultés rencontrées diminuent en fonction de l'augmentation de la confiance en soi, des remarques en fin de questionnaire font souvent référence au stress vécu pendant l'année et les examens. Lors du passage de CM2 en $\mathrm{CM} 3$, on peut être étonné de la réduction statistiquement significative des scores d' " analyse-synthèse " et de "mémorisation par appropriation " et de l'absence d'utilisation accrue de l'outil " apprentissage en profondeur ". Cette observation confirme le vécu des examinateurs à l'issue de la CM3 et traduit probablement une augmentation importante du nombre d'enseignements et l'absence de coordination manifeste entre les titulaires.

L'analyse des résultats doit impérativement tenir compte des conditions « locales». Ainsi, il n'existe pas de limitation à l'inscription en première année : aucune sélection n'oppose quelque barrière que ce soit à l'admission. De surcroît, les études secondaires ne sont pas sanctionnées par une épreuve normalisée de type baccalauréat. Les étudiants portent donc les forces et les faiblesses de leur formation antérieure et de leur situation socio-économique. Certains étudiants sont ainsi décontenancés par un discours universitaire assez éloigné de leur capital culturel propre, ce qui constitue un facteur de démotivation ${ }^{8,9}$. Néanmoins, malgré le caractère dissemblable des filières, certaines tendances se rapprochent de ce qui ressort d'études réalisées dans d'autres contextes ${ }^{2,10,11}$.

Il ne fait nul doute qu'outre l'intérêt propre des présentes données, celles-ci constituent des arguments précieux susceptibles de venir a l'appui d'une éventuelle réforme de la troisième année, qui tendrait à réduire le nombre d'épreuves et à susciter une étude plus synthétique. De surcroit, les résultats de l'enquête permettent de cibler plus efficacement la remédiation à mettre en œuvre dès le début des études. En outre, compte tenu de l'absence de sélection à l'entrée des études et du caractère résolument universitaire de l'enseignement dispensé (centré sur le questionnement, la recherche, l'excellence), il est impératif d'envisager une initiation aux pratiques universitaires et aux stratégies d'apprenti- 


\section{Recherche et Perspectives}

ssage, susceptible de renforcer chez les étudiants les pratiques métacognitives ${ }^{10,12,13,14}$.

La comparaison des résultats obtenus respectivement chez les étudiants de CM1, de BM1 et d'E/A1 montre que le recours aux stratégies de " mémorisation " paraît identique. Cependant, beaucoup d'outils évalués, qu'ils se rapportent à des comportements cognitifs, métacognitifs ou affectifs, sont plus faiblement utilisés par les étudiants de BM1 et d'I/A1 que par ceux de CM1 (le nombre de difficultés rencontrées est quant à lui plus grand pour les étudiants de BM1 et d'I/A1). La différence observée au niveau de l'outil « exemplification » au profit des étudiants d'I/A1 est en rapport avec la finalité directement perceptible des études concernées et le transfert aisé des connaissances acquises. L'ensemble de ces données se traduit par un faible score de l'« item » lié à la réussite scolaire pour les étudiants de BM1 et d'I/A1.

Les données qui ont été acquises dans le cadre de cette étude, et tout particulièrement celles qui résultent de la comparaison des filières, présentent l'indéniable intérêt de soutenir l'idée que les publics étudiants en sont différents et que l'approche pédagogique doit s'y concevoir de manière spécifique à chacune d'elles. Il devrait en être de même pour les stratégies de remédiation, qui doivent tenir compte des particularités de chacun des groupes. Certains groupes étudiés sont en réalité assez hétérogènes. L'enseignement supérieur hors université accueille d'une part des "primo-arrivants", venus de milieux socioéconomiques pour lesquels des études « courtes » et à visée professionnelle représentent une promotion sociale raisonnable ${ }^{15}$, mais aussi des étudiants redoublants (parfois d'origine variée) pour lesquels l'expérience acquise est facteur indéniable de réussite, comme c'est du reste également le cas dans la filière universitaire. Les remarques émises à la suite de la comparaison antérieure et relatives à la nécessité d'une remédiation ciblée et d'une initiation aux stratégies d'apprentissage gardent leur validité mais doivent être adaptées au public concerné.

L'utilisation dans un groupe étudié d'étudiants provenant de cohortes historiques différentes limite l'effet de cohorte ; les résultats obtenus au fil des trois candidatures sont le reflet d'une évolution générale et non celles d'étudiants en particulier.

Lorsque nous avons demandé aux étudiants s'ils voulaient participer au questionnaire, nous avons été surpris par le nombre d'étudiants enthousiastes. La possibilité de rester anonyme a vraisemblablement favorisé la sincérité des réponses données par les étudiants. Ils nous ont fait part de l'intérêt qu'ils portent aux stratégies d'apprentissage à adopter ou à découvrir lors de leur étude. Mais ils ne semblent pas avoir d'idées claires sur leurs propres stratégies.

Concernant l'étude dans son ensemble, nous sommes conscient du caractère qualitatif des résultats et nous ne considérons pas ceux-ci comme normatifs. Les limites méthodologiques de notre travail sont celles de l'étude princeps, à savoir qu'il s'agit des stratégies d'apprentissage rapportées par les étudiants, que les questions concernant tous les outils sont à réponse fermées et enfin que l'échantillon d'étudiants est relativement faible?

\section{Conclusion}

La connaissance des outils utilisés peut amener l'étudiant à mieux comprendre son comportement devant l'étude, à s'adapter aux différentes situations d'enseignements. Une meilleure connaissance de ses stratégies métacognitives pourrait aider l'étudiant dans la gestion de son stress.

La connaissance de certaines lacunes dans l'apprentissage des étudiants pourrait aussi aider l'enseignant à modifier ces habitudes. Des disparités importantes dans l'échec selon les matières poussent à la réflexion. Le questionnement de l'étudiant à propos de sa réussite est une voie à suivre. Les grandes différences observées entre les étudiants de première année de graduat et de médecine nous renforcent dans l'idée que les stratégies pédagogiques utilisées lors de l'enseignement pour ces deux groupes doivent rester spécifiques du type d'enseignement, ce qui n'exclut évidemment pas des possibilités de recoupement dans certaines circonstances ${ }^{16}$.

\section{Remerciements}

Les auteurs remercient vivement Madame G. Buscarlet, directrice de l'Ecole d'Infirmier(e)s annexée à l'ULB, ainsi que le Professeur J.-L. Wolfs, professeur à la faculté des sciences psychologiques et de l'éducation de l'ULB.

\section{Contributions}

Nathalie Vanmuylder et Patrick Salvia ont rédigé et dépouillé le questionnaire. Nathalie Vanmuylder et Stéphane Louryan ont rédigé l'article. Patrick Salvia a réalisé les tests statistiques. Marcel Rooze était Doyen de la faculté de médecine et a encouragé l'initiative de l'enquête. Françoise De Broeu était chef de travaux de l'unité d'enseignement et de recherche de biologie médicale à la Haute Ecole Francisco Ferrer et a facilité la récolte des données. 


\section{Références}

1. Ausubel DP, Novak JD, Hanesian H. Educational psychology. A cognitive view. $2 d$ edition. New York: Holt, Rinehart and Winston, 1978.

2. Boulet A, Savoie-Zajc L, Chevrier J. Les stratégies d'apprentissage à l'université. Sainte-Foy: Presses de l'Université du Québec, 1996.

3. Pressley M, Levin JR, Ghatala ES. Memory strategy monitoring in adults and children. Journal of verbal learning and verbal behavior 1984;23:270-88.

4. Gagné E.D. The cognitive psychology of school learning. Boston: Little Brown, 1985.

5. Pressley M, Levin JR, Ghatala ES. Children metamemory and the teaching of memory strategies. in: ForrestPressley DL et al. (Eds): Metacognition, Cognition and human performance. New York (NY) : Academic Press, 1985:111-53.

6. Collectif. Un pôle...pourquoi ? Bruxelles : Editions du Céfal, 2003.

7. Wolfs J-L. Méthodes de travail et stratégies d'apprentissage. Bruxelles : De Boeck Université, 1998.

8. Bourdieu P, Passeron JC. La reproduction. Paris : Editions de Minuit, 1970.
9. Louryan S, Thys-Clément F, (Eds.). Enseignement secondaire et enseignement universitaire. Quelles missions pour chacun? Bruxelles : Editions de l'Université de Bruxelles, 1999.

10. Côté DJ, Bellavance C, Chamberland M, Graillon A. Un programme pour aider les étudiants en médecine à développer leurs stratégies d'apprentissage. Pédagogie Médicale 2004;5:95-102.

11. Lahtinen $V$, Lonka $K$, Lindblom-Ylänne $S$. Spontaneous study strategies and the quality of knowledge construction. Br J Educ Psychol 1997;67:13-24.

12. Noël B. La métacognition. Bruxelles : De Boeck Université, 1991.

13. Frenay $M$, Noël B, Parmentier P, Romainville $M$. L'étudiant-apprenant. Bruxelles: De Boeck Université, 1998.

14. Romainville M. Savoir parler de ses méthodes. Métacognition et performance à l'université. Bruxelles: De Boeck Université, 1993.

15. Boudon R. Linégalité des chances. Paris : Armand Colin, 1979.

16. Thys-Clément F, Verrept $M$, Louryan S, (Eds.). Université, hautes écoles : quelles synergies? Bruxelles: Editions de l'Université de Bruxelles, 2001.

Manuscrit reçu le 16 août 2004; commentaires éditoriaux formulés aux auteurs le 18 février et le 28 novembre 2005; accepté pour publication le 30 novembre 2005. 